\title{
Research on Dynamics Model for Web Comment
}

\author{
Qiumei $\mathrm{Pu}^{\mathrm{a}}$, Hong Zhang ${ }^{\mathrm{b}}$ \\ Minzu University of China, Department of Information Engineering, China \\ alunar_sea@163.com, ${ }^{\mathrm{b}}$ Zhang hong @163.com
}

\begin{abstract}
View of web comment is affect the potential consumers purchasing decisions and the impact on the final results of the growing corporate sales significantly, thus the evolution of the view is an important study more and more. Based on complex network, we build dynamics model in web comment mode to analyze the basic characteristics of review evolution. Based on simulation analysis, we provide suggestions on effective web comment management for enterprises.
\end{abstract}

Keywords-complex network; WEb comment; evolution; simulation analysis.

\section{INTRODUCTION}

With the development of communication technology and electronic commerce, web comment gradually becomes popular in public. It helps to exchange information about product and service quality among consumers and provides the basis for doing research on online consumer behavior and decision-making. The premise and foundation for optimizing web comment management methods is to analyze the opinion interaction and evolution mechanism. And opinion dynamics model is one of the analysis methods. Based on the basic physics principles, we established the dynamics model, and research on the public opinion evolution through model simulation.

In the last ten years, physicists have begun to actively commit to public opinion dynamics, and many models have been designed. Our concern is that caused widespread concern in the physical literature and noted the similarities and differences between the models. The voter model, majority models, based on the theory of social impact grinding (social impact theory) model, Sznajd and bounded confidence model (bounded confidence models)[1][2]. This article focuses on the analysis of the model for the confidence model (DeffuantModel and $\mathrm{KH}$ models) [6].

Deffuant Model $\mathrm{KH}$ models are finite and the trust model (Bounded Confidence), i.e. the presence of a confidence interval (or called threshold); only the gap between views (point here is $\in[0,1]$ ) will be within the $s$ affect each other.[6]

At present, the complex network is widely adopted in opinion dynamics model. However, it is quite different from web comment. Therefore, it needs to construct the opinion dynamics model suitable for web comment mode.
This paper is focused on constructing the opinion dynamics model applying to web comment environment, using this model to analyze the web comment management strategies in JD (abbreviation of JingDong Mall) and providing theoretical support for web comment management on e-commerce platform.

\section{RELATED WORKS}

Literature [3] analysis the related factors of relationship between them by dynamic evolution rule, and make a reasonable experiment by the use of advanced information technology to deal with the opinion information.

Literature [4] we proposed a multi-agent opinion dynamics model for Online Mass Incident, in which selecting likely-minded neighbors or opponents is decided by individual relevance. Drawing on the research findings of social psychology, Numerical simulations show that, high individual relevance favors appearance of large clusters and lessening of extremism, which is qualitatively agree with suggestions of sociologists and actual practice of governments.

Morarescu [5] study a class of discrete-time multi-agent systems modeling opinion dynamics with decaying confidence. Heconsiders a network of agents where each agent has an opinion. At each time step, the agents exchange their opinion with their neighbors and update it by taking into account only the opinions that differ from their own less than some confidence bound. This confidence bound is decaying: an agent gives repetitively confidence only to its neighbors that approach sufficiently fast its opinion. In this paper, he analyze this opinion dynamics model and show that communities correspond to asymptotically connected components of the network

The purpose of our paper is to apply opinion dynamics model to e-commerce and our method is to make autocorrelation function program of electronic products to reflect the user's potential intent. Moreover, evolution of web comment can realize the analyzing other kinds of intervention strategies.

The rest of the document is structured as follows. In section 2, we resume the related works and summarize opinion dynamics models method in some fields. In section 3 , we put forward how to create evolution of web comment and describe the evolution process. In section 4 , we describe the simulation analysis based on the opinion 
dynamics model. In section 5, we present conclusions and put forward a few shortcomings existing in our research.

\section{EVOLUTION MODEL IN WEB COMMENT MODE}

\section{A. Selection of parameters}

The basic parameters should be determined in constructing the opinion dynamics model in web comment mode. On the basis of Deffuant model and $\mathrm{KH}$ model, four parameters are included in this model, such as opinion difference threshold $\varepsilon$, convergence parameter $\mu$, opinion release probability $\mathrm{e}$ and the maximum number of reference reviews $\mathrm{m}[6]$.

1) Opinion difference threshold

Opinion difference threshold $\varepsilon$ represents the maximum difference in opinion acceptation. In web comment, one individual has access to views coming from the strangers and may not trust the point of view which is quite different from his own. Therefore, it is necessary to take the confidence interval into consideration.

In this model, $\varepsilon$ has great influence in final view quantity, but less in relaxation time, which is the time required reach a final consensus for an opinion.

\section{2) Convergence parameter $\mu$}

Convergence parameter $\mu$ represents the individual trust degree for others' views. The bigger the parameter $\mu$, the greater one can be influenced by new views when the views are updated. In this model, $\mu$ has great influence in relaxation time and the final opinion formation.

3) Opinion release probability e

Opinion release probability $\mathrm{e}$ is the probability of each individual releasing new comment at every moment, which reflects the individual activity.

4) Maximum number of reference reviews $m$

Maximum number of reference reviews $m$ limits the number of reviews every individual can see at every moment.

5) The influence of initial opinion distribution

Both the distribution and average value of initial opinion have influence in the final opinion's distribution and the evolution process.

\section{B. Opinion dynamics model in JD}

This study randomly selected a variety of one month in 2000 sales of electronic products review for validation in JD. The selection criteria of product are as follows:

(1)When consumers buy electronic products generally more concerned about product reviews, that is to say the comment would have a significant impact on their view and purchase behavior.

(2)There have been no price promotions. This study was conducted excluding the short-term commodity price promotions in order to avoid the purchase price and the number of comments fluctuations caused.

(3)The products sales amount is more than one thousand. From the actual data collection situation, higher volume sales of goods is very possible case records were incomplete; while sales of low and difficult to provide sufficient data. Therefore, this study tries to select sales of large goods to ensure data integrity in the premise.

From the verification results, opinion dynamics model obtained in this study may better reflect the reality of the environment JD reviews generation mechanism. As the verification results can be shown, the opinion dynamics model in JD can represent the review generation mechanism in actual JD environment. The following is description of figure-1.
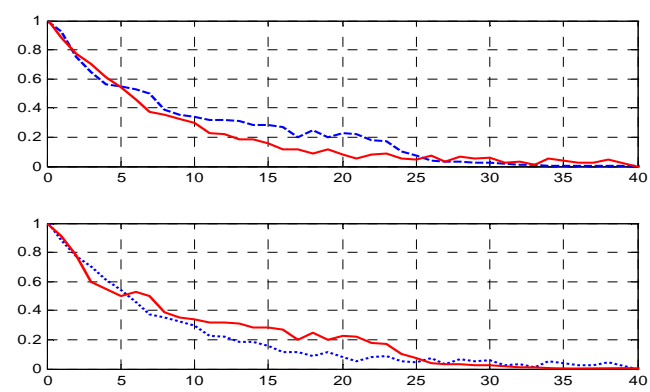

Figure 1. The review generation mechanism in actual JD environment.

The dashed line represents the simulation purchase amount and the solid line represents the actual purchase amount.

It is very necessary to adjust the basic opinion dynamics model in combination with the opinion interaction mode in specific circumstances. It will report about making adjustments of this model and analyzing review management strategies in JD.

The group's openness is the greatest difference between the opinion interaction way in JD and the basic theory model. In the basic theory model, the individuals participating in opinion interaction are fixed without change of personnel. In JD, the individuals involved at every moment are different. Another difference lies in the synchronization while browsing and releasing a comment. In the basic theory model, browsing and releasing comments happened at the same time, while in JD it will have a little delay.

Two validation methods are generally used in traditional opinion dynamics model research. One is to compare the final opinion distribution in simulated and actual conditions. The other one is to compare the autocorrelation function of opinion evolution process in these two conditions. Autocorrelation function can reflect the correlation degree of the same sequence at different moment, which is of great help for comparison in different internal mechanisms. This paper adopts the second method to validate the correlation of the evolution process in simulated and actual conditions and conducts the preliminary validation for opinion dynamics model in JD.

\section{SIMULATION ANALYSIS BASED ON THE OPINION DYNAMICS MODEL}

Based on the preliminary validation of the opinion dynamics model, analyze the review sorting and batch 
display methods to study the different review management strategies' impact on community opinion evolution.

Although various types of comments sorting method has been widely used on many sites, but there needs to be further analyze different perspectives on consumer information sorting methods affect the evolution of the characteristics and effects. In this study, after a preliminary verification of public opinion dynamics model, discussed the impact on consumers view comments sorting method and the results of the evolution of a variety of situations.

For each parameter configuration, simulate the review evolution in different sorting methods. Select the first two scenarios to display the results, as shown in figure-2.
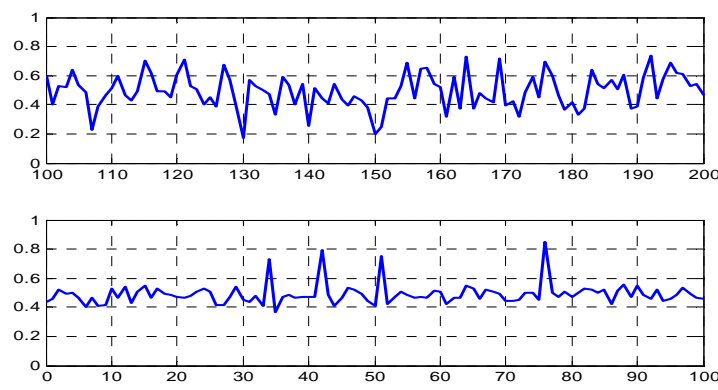

Figure 2. Review evolution process in different scenarios

(X-axis: time. Y-axis: the average value of consumer opinion at every moment)

Each parameter combinations can be simulated in real commodity characteristics, and thus can be used for analysis of the relevant point of view of its evolution and results.

Batch display can avoid the negative effect the extreme views made on customers when the number of comments is small. To simulate the situation using this method, adjust the rules. When the amount of the new reviews is below a certain threshold, temporarily don't show these comments. When the amount of the new reviews (including the new comments in the cache) reaches the threshold, all new reviews will be added to the review list.

According to the experimental results, adopting the batch display method has little influence in the final results of opinion evolution, such as the average value of the final opinion, purchase quantity, review quantity, but much in opinion evolution.
The opinion evolution is stable with the decreasing influence of early extreme views. It reaches a certain threshold value will the first batch of comments be displayed, which dilute the influence of the early extreme reviews to the customers to a great extent and guarantee the stability of the experiments results.

\section{V.FUTURE WORKS}

In this paper, the basic model and the adjusted model in $\mathrm{JD}$, not only can be used to analyze the nature of the communication process, but also can provide foundation platform for analyzing other kinds of intervention strategies. At the same time, we can consider the situation with multiple products, stores and purchases. This paper only considers the simplest situation with a single product, a single store and a single purchase, which may cause the relatively limited review and purchase quantity. But in reality, the web comment platform is more complicated. For example, there are a variety of products and stores in JD platform at the same time, and the consumers can determine the subsequent purchase and reviews according to their own needs and the previous experience. In condition of multiple products, stores and purchases, the volatility of consumers' reviews on commodity is likely to have more complicated and obvious impact on purchase behavior.

\section{REFERENCE}

[1] Sznajd Weron, K. and J. Sznajd, Opinion evolution in closed community. International Journal of Modern Physics C; 2000. 11(06): p. 1157-1165.

[2] Hegselmann, R. and U. Krause, Opinion dynamics and bounded confidence: models, analysis and simulation. Journal of Artificial Societies and Social Simulation, 2002. 5(3).

[3] Du, Jin ; Du, Yanhui; A Dynamics Model of Opinion Information System, ISCCC2013, Page(s): 6 - 12

[4] [4]XiaMengSi;YunLiu;HuiCheng;YanChao Zhang;An opinion dynamics model for Online Mass Incident; ICACTE2010Page(s): V596-V599

[5] Morarescu, I.C. ; Girard, A. Opinion Dynamics with Decaying Confidence: Application to Community Detection in Graphs Automatic Control, IEEE Transactions on Volume: 56 , Issue: 8 2011, Page(s): 1862 - 1873

[6] Fortunato, S., Universality of the Threshold for Complete Consensus for the Opinion Dynamicss of Deffuant et al. International Journal of Modern Physics C: Computational Physics \& Physical Computation, 2004. 15(9): p. 1301-1307. 